\title{
EL PENSAMIENTO CONSTRUCTIVO PARA LA ENSEÑANZA DEL EMPRENDIMIENTO: CASO COLEGIO JOHN DAVISON ROCKEFELLER QUITO-ECUADOR
}

\section{CONSTRUCTIVE THINKING TO THE TEACHING OF ENTREPRENEURSHIP: CASE SCHOOL JOHN DAVISON ROCKEFELLER QUITO-ECUADOR}

\author{
Nelson Iván Benalcázar Atiencial, Luis Alfonso Blanco Benitez²
}

\begin{abstract}
Resumen
Este estudio se realizó con el propósito de analizar el desarrollo del pensamiento constructivo y la enseñanza del emprendimiento en colegios de educación media especificamente como caso de estudio el colegio John Davison Rockefeller ubicado en la ciudad de Quito. Se tomó como muestra 45 alumnos de los cuartos, quintos y sextos cursos, en edades de 15 a 19 años, quienes respondieron el cuestionario CTI aplicado para este efecto, lo cual permitió analizar el desarrollo emocional, la salud física y ciertos valores como la responsabilidad aspectos que van adquiriendo en su vida personal y su impacto en los métodos de educación en los estudiantes de nivel secundario para preparar proyectos creativos e innovadores de emprendimiento.
\end{abstract}

Palabras clave

Pensamiento constructivo, creatividad, innovación, enseñanza, emprendimiento.

Clasificación JEL: |25, 043

\begin{abstract}
This study was carried out with the purpose of analyzing the development of constructive thinking and the teaching of entrepreneurship in middle education schools, taking as a case of study the John Davison Rockefeller College, located in the city of Quito. The sample were 45 students from the $4^{\text {th }}, 5^{\text {th }}$ and 6 th courses, within the ages of 15 to 19 years old, who responded to the CTI questionnaire applied to this effect, allowing us to analyze the emotional development, physical health and certain values, like the liability aspects they are acquiring in their personal lives and their impact on the education methods in the students of secondary level in order to prepare creative and innovative entrepreneurial projects.
\end{abstract}

Keywords

Constructive thinking, creativity, innovation, education, entrepreneurship.

JEL Classification: 125, 043 


\section{Introducción}

Según Seymour Epstein, el pensamiento constructivo como herramienta se enfoca en la parte emocional de los individuos y permite comparar de qué manera influyen las emociones y sus experiencias en su vida diaria. El propósito del estudio realizado tiene como fin analizar cómo afectan las emociones y sus experiencias en el proceso de emprendimiento a los estudiantes de educación media.

Además, si la institución educativa ha construido una cultura de emprendimiento sustentada en valores, principios y emociones que impulsen en los estudiantes el sentido de pertenencia y motivación para lograr proyectos de emprendimiento innovadores y creativos.

Para efectos de esta investigación se analizó otros instrumentos de exploración cognitiva, se seleccionó el CTI, porque es un instrumento aplicable a jóvenes y adultos, además considera que las personas aprenden de sus experiencias vividas y hace referencia a la capacidad para reconocer sus emociones y sentimientos en la solución de problemas y en el logro de las metas y objetivos que cada individuo se propone, acciones indispensables para el proceso de emprendimiento.

Encontrar un empleo, hoy resulta cada vez más difícil, esto influye en las condiciones económicas y de desarrollo de los jóvenes; qué se puede hacer entonces, infundir en los alumnos el deseo de convertirse en empresarios, que mantengan la visión de desarrollar sus propios negocios, el emprendimiento puede ayudar no solo a la reactiva-

\section{Marco teórico}

El pensamiento constructivo tiene como sistema primario la inteligencia emocional, racional y práctica; es el resultado de los constructos entre las inteligencias menores ción económica sino a la creación de fuentes de trabajo (Hernández \& Arano, 2015).

El emprendimiento se le considera como la habilidad, los conocimientos y la experiencia que tienen las personas para construir una buena idea de negocios, estas son aptas para ingeniarse ideas innovadoras y creativas. La cultura emprendedora está ligada con la innovación y la investigación, lo que busca es crear o transformar un producto o servicio en algo más atractivo y con mayor ventaja competitiva (Hernández Rodríguez \& Arano Chávez, 2015).

Según los autores Viloria \& Rodríguez (2019) llegar a concretar una idea de negocios en una empresa es una manera significativa de creer en uno mismo y de las capacidades desarrolladas como emprendedor, estimuladas por factores como la creatividad, la proactividad y el deseo de mejorar su condición económica y social.

Los autores Hernández \& Arano (2015) consideran que desde tiempos antiguos la cultura emprendedora ha existido, así como siempre existió la necesidad de innovar para poder subsistir en un mundo cada vez más cambiante, el hombre y la sociedad han buscado satisfacer sus necesidades y mejorar su calidad de vida, a través del emprendimiento.

En la actual sociedad del conocimiento, se considera una época donde el individuo posee diversas tecnologías de comunicación e información para mostrar sus habilidades, capacidades, propuestas creativas, tanto en productos como en servicios con resultados innovadores (Viloria \& Rodríguez, 2019).

como la experencial y la racional, elementos que solidifican al pensamiento constructivo (Muñoz, 2010). Luego los estudios realizados en este campo nos mencionan que el gran 
pensador constructivo es mucho más eficaz que el pensador intelectual, así lo dice Seymur Epstein autor del Pensamiento constructivo (1998), dice que las personas que cuentan con un alto pensamiento constructivo se relacionan con una gran posibilidad de tener éxito en su vida personal, no así las personas que tienen una baja inteligencia emocional, ya que tienden a ver el mundo que les rodea de una manera negativa, minimizando el desarrollo de sus capacidades para lograr sus metas y objetivos personales (Chavéz Becerra, Contreras Gutiérrez, \& Velásquez Ortiz, 2013).

El pensamiento constructivo se deriva de la experiencia vivida por el individuo, se relaciona con el éxito en las diferentes actividades que realiza el ser humano como son: el trabajo, la salud, la parte espiritual, la parte emocional, las relaciones personales y la satisfacción enfocada en la felicidad (Muñoz, 2010).

El concepto del pensamiento constructivo, según la teoría cognitiva experiencial formulada por Epstein (1994), es una forma de aprendizaje sustentado en la experiencia vivida considera que la gente se adapta a su entorno y dirige su conducta hacia él (García, 2005).

El Inventario de Pensamiento Constructivo (CTI), se fundamenta en la experiencia del individuo, su aplicación como herramienta ayuda a: estudiar, evaluar y predecir aquellas experiencias vividas y aquellas a las cuales se adapta un individuo y que son necesarias para tener éxito en la vida (Epstein, 2012), consiste en un test que permite evaluar el pensamiento constructivo o inteligencia experimental y su relación directa con la inteligencia emocional (IE) por medio de "la evaluación de los pensamientos diarios automáticos, constructivos o destructivos, que las personas tienen" (Epstein, 1998).

El análisis del pensamiento constructivo, según Epstein (1998) proporciona tres niveles de puntuaciones:
- Escala global de pensamiento constructivo,

- Escalas principales son seis: constructivo (afrontamiento emocional y afrontamiento conductual) y destructivo (pensamiento mágico, pensamiento categórico, pensamiento esotérico y optimismo ingenuo),

- Facetas: son quince que describen modos específicos, cómo pensar positivamente, dejar de aferrarse a acontecimientos desafortunados del pasado, clasificar a la gente o a los hechos de modo categórico o pensar en formas que favorecen o interfieren en una actuación eficaz como: autoestima, ausencia de sobre generalización negativa, ausencia de hipersensibilidad, experiencias desagradables, pensamiento positivo, orientación a la acción, responsabilidad, pensamiento polarizado, suspicacia, intransigencia, creencias paranormales, pensamiento supersticioso, optimismo exagerado, pensamiento estereotipado, ingenuidad (CTI, 2015).

La aplicación del (CTI) es una prueba que evalúa el pensamiento constructivo y otros aspectos de la inteligencia emocional (IE), como son la motivación, la inteligencia práctica, la fuerza del yo, el control apropiado de las emociones, la habilidad social y la creatividad (Epstein, 1998).

Por consiguiente, la inteligencia experiencial se refiere a generar pensamientos que funcionan por asociaciones en vez de por lógica, los que favorecen a la solución de problemas, creando una fuerte relación entre aquellos acontecimientos que tienen una fuerte carga emocional asociada con la experiencia afectiva o emocional (García, 2005).

Según García (2005), las personas cuentan con dos tipos de inteligencia: una inteligencia racional y una experiencial, esta última pueden ser considerada como un conjunto de habilidades básica que las dis- 
tingue y que contribuyen, por diferentes caminos, al éxito en la vida.

Epstein (1998) sostiene que se puede entender el pensamiento constructivo como "esquemas mentales de representación de la realidad que orientan la percepción, las emociones y acciones hacia la solución de problemas y la consecución de metas".

Por lo tanto, el pensamiento constructivo es "un instrumento de evaluación de pensamientos diarios constructivos o destructivos que permiten predecir reacciones adaptativas, así como toda una serie de habilidades que influirán en el éxito académico, en la satisfacción en el trabajo, en la capacidad de afrontamiento del estrés, en el ajuste emocional o el bienestar físico y mental" (López Sánchez, Jiménez Torres, \& López Núñez, 2011).

Este tipo de pensamiento se orienta a considerar que el individuo al procesar información de los acontecimientos, emociones y eventos que acontecen en el día a día, produce la interpretación inconsciente y específica de percibir la realidad y su comportamiento se debe a las experiencias vividas (Contreras, Chávez, Aragón, \& Velásquez, 2011).

El pensamiento constructivo se enfoca en la capacidad para reconocer las emociones, sentimientos y la personalidad. Se fundamenta en aquellos pensamientos que aparecen en nuestra mente en forma voluntaria y que direccionan nuestra vida para lograr las metas y solucionar los problemas cotidianos (Contreras, Chávez, Aragón, \& Velásquez, 2011).

Álvarez \& Camargo (2007) consideran que las experiencias vividas contribuyen a desarrollar el pensamiento constructivo y no la preparación académica. Consecuentemente, la inteligencia experiencial se relaciona más con el pensamiento constructivo que la racional.

En la sociedad actual, las relaciones entre sus integrantes y el intercambio de in- formación, se encuentran influenciadas por el uso de sistemas de información y comunicación. Estos adelantos científicos y técnicos aplicados en la formación de personas permiten aprender, desaprender y reaprender (Rodríguez Ramírez, Delgadillo Salgado, $\&$ Torres Trejo, 2018).

Dentro del proceso de enseñanzaaprendizaje, la educación actual contempla aspectos teóricos como prácticos, una corriente para este fin es el constructivismo, que sostiene que el conocimiento es una asimilación activa de la realidad en estructuras que van de las más simples a las complejas (Rodríguez Ramírez, Delgadillo Salgado, \& Torres Trejo, 2018).

El constructivismo sostiene que el docente requiere construir un ambiente de confianza con el alumno, atando el contenido académico con las experiencias y vivencias de ambos actores y su aplicación en la práctica (Rodríguez Ramírez, Delgadillo Salgado, \& Torres Trejo, 2018).

Por lo tanto, en la actualidad las inclinaciones pedagógicas han tomado como bandera al constructivismo debido a que al estudiante le permite comprender los procesos y principios de forma activa (Rodríguez Ramírez, Delgadillo Salgado, \& Torres Trejo, 2018).

Uno de los desafíos a los que se enfrentan los programas de formación académica, es conseguir que los estudiantes desarrollen habilidades y conocimientos que les permitan obtener resultados profesionales excelentes en el futuro (Puebla \& Termiño, 2018).

En ocasiones la formación es criticada por ser excesivamente teórica, poco creativa e innovadora y alejada del mundo real (Puebla \& Termiño, 2018).

La educación actual exige que utilicemos metodologías de formación innovadora y creativa, más apegada al mundo empresarial. Al mismo tiempo, en su aplicación, estas metodologías deben ser integradoras de forma que potencien la coherencia de las 
diferentes materias dentro de un plan de estudios integral (Puebla \& Termiño, 2018).

No se promueve la habilidad de crear o innovar, porque no se conoce adecuadamente cómo se puede aprender y desarrollar. La primera condición para ser creativo es desarrollar la habilidad de pensar y actuar, para modificar o transformar lo que conocemos y tener la libertad de cuestionar lo que es aceptado oficialmente. (Toca, 2017)

Para Toca (2017), la innovación se considera como la modificación de algo previamente creado, sea una idea, un diseño o un producto.

Dos competencias que deben incorporarse en los currículos académicos para la formación profesional son la creatividad e innovación, estas deben desarrollar todas las personas, sin importar el nivel de educación y el tipo de formación, además, son necesarios para el desempeño académico y laboral (Hernández \& Alvarado, 2014).

El desing thinking (DT) es una técnica que estimula la creatividad y la innovación, que se está aplicando en las organizaciones como una forma estratégica de innovar, el proceso consiste en empatizar con las necesidades de los clientes, buscar información, idealizar, crear un prototipo y testear (Pelta, 2009).

¿Cómo aprenden los estudiantes? Esta interrogante plantea un cambio de mirada, un giro drástico en la educación, pues pasa de un proceso centrado en la enseñanza a un proceso centrado en el aprendizaje. El conocimiento lo construye el alumno a través de procesos cognitivos internos, de su relación con los demás y de la realidad que lo rodea (Huerta, 2019).

La satisfacción de las ilimitadas necesidades humanas son una fuente inagotable de oportunidades de negocio, bajo este contexto, el emprendimiento surge como una forma de aportar con soluciones efectivas para la reactivación económica (Marulanda Montoya, Correra Calle, \& Mejía Mejía, 2009).
El emprendimiento es un tema relevante de gran importancia no solo para la economía del país, sino por la necesidad de muchas personas y organizaciones de ver que el personal sea capaz de emprender e iniciar sus propios negocios, y pasar de ser empleados a ser empleadores (Gutiérrez 0, Asprilla M, \& Gutiérrez L, 2016).

Dentro de las competencias más importantes que debe contar un emprendedor es la creatividad, "hacer cosas nuevas o hacerlas diferentes creando valor" (Marulanda Montoya, Correra Calle, \& Mejía Mejía, 2009).

El espíritu emprendedor actúa para que el emprendedor pueda mejorar y desarrollar nuevos negocios para satisfacer las necesidades de la población, permitiendo incrementar la productividad y generar la mayor parte del empleo (Zamora, 2018).

El proceso del emprendedor depende de algunos factores tales como: las condiciones personales de los emprendedores, del mercado, recursos financieros e información, así como la intervención de los gobiernos a través de programas y proyectos públicos para apoyar al proceso emprendedor (Zamora, 2018).

Según los autores Hernández Rodríguez \& Arano Chávez (2015) uno de los problemas a los que se enfrentan los jóvenes que egresan de las universidades es la falta de oportunidades para conseguir empleo.

Es por ello la importancia de construir una cultura emprendedora entre los estudiantes de niveles secundarios y universitarios con la intensión de despertar en ellos el deseo de transformarse en empresarios (Hernández Rodríguez \& Arano Chávez, 2015).

El contexto actual la sociedad del conocimiento tiene su fundamentación en la necesidad de personas con creatividad, innovación y emprendimiento, por otra parte los procesos educativos deben centrarse en el desarrollo de esas competencias (De la Fuente Arias, Vera Martínez, \& Cardelle-Elawar, 2012). 


\section{Metodología}

La investigación es cualitativa de carácter descriptivo y consiste en la aplicación del test CTI o Inventario de Pensamiento Constructivo, que es una prueba para adolescentes y adultos, permite la evaluación del pensamiento constructivo y otros aspectos de la inteligencia emocional (IE), como son la motivación, la inteligencia práctica, la fuerza del yo, el control apropiado de las emociones, la habilidad social y la creatividad, el mismo que se puede realizar de forma individual o colectiva, por lo tanto se refiere a la habilidad de generar pensamientos que favorecen la solución de problemas de la vida diaria. Se consideró a los 45 estudiantes de educación media de cuartos, quintos y sextos cursos del colegio John Davison Rockefeller, 29 hombres y 16 mujeres en edades comprendidas entre los 15 a 19 años.

\section{Resultados}

La población estudiada se encuentra conformada por estudiantes en edades de 15 a19 años; 29 hombres y 16 mujeres, su edad promedio fue de 17 años. De acuerdo al estudio de pensamiento constructivo, cuando se trata de una persona la interpretación está dada para establecer puntos específicos que sobresalgan de su perfil, diagnosticar su tipo de pensamiento individual y con ello constituir su constructividad con respecto al medio. Cuando se trata de una institución en este caso educativa la interpretación cambia, se debe establecer un diagnóstico general para determinar cómo se encuentran los estudiantes en el campo del pensamiento constructivo y compaginar con sus otros perfiles para tomar decisiones con respecto a tres ejes, institución-entorno-estudiantes y de acuerdo con ello mejorar, mantener o
Para analizar los perfiles de CTI (Pensamiento Constructivo), se aplicó el instrumento que está enfocado a la parte emocional y personal de los estudiantes, con el fin de conocer cuáles son los puntos fuertes, débiles, actitudes y aptitudes de cada uno de ellos y su orientación hacia la creatividad, la innovación y el emprendimiento.

Este Inventario o test CTI está conformado por 108 preguntas en escala Likert, de cinco puntos en función del grado de: totalmente en desacuerdo, en desacuerdo, neutral, en acuerdo y totalmente de acuerdo, en cada una de las afirmaciones.

Con el apoyo del programa estadístico SPSS, se procesó la información para luego realizar la interpretación y análisis de los resultados obtenidos.

superar puntos de constructivismo para su mejor desarrollo. Esto se logra con la recopilación de datos de todos los involucrados en el estudio; $y$, de esta manera manejar rasgos que permitan establecer puntos que sean más críticos y que necesitan ser tratados para hacer de este medio un espacio altamente productivo con base en el pensamiento constructivo, la creatividad, innovación y el emprendimiento.

En el cuadro 1, se puede apreciar los resultados del test de Inventario de Pensamiento Constructivo aplicado a los estudiantes de educación media y la tendencia general.

En el cuadro 2 se aprecia la tabulación del primer cuadro, considerando los resultados más altos de cada pregunta (opción A, B, C, D०E). 
Cuadro 1. Tendencias generales por edad de pensamiento constructivo

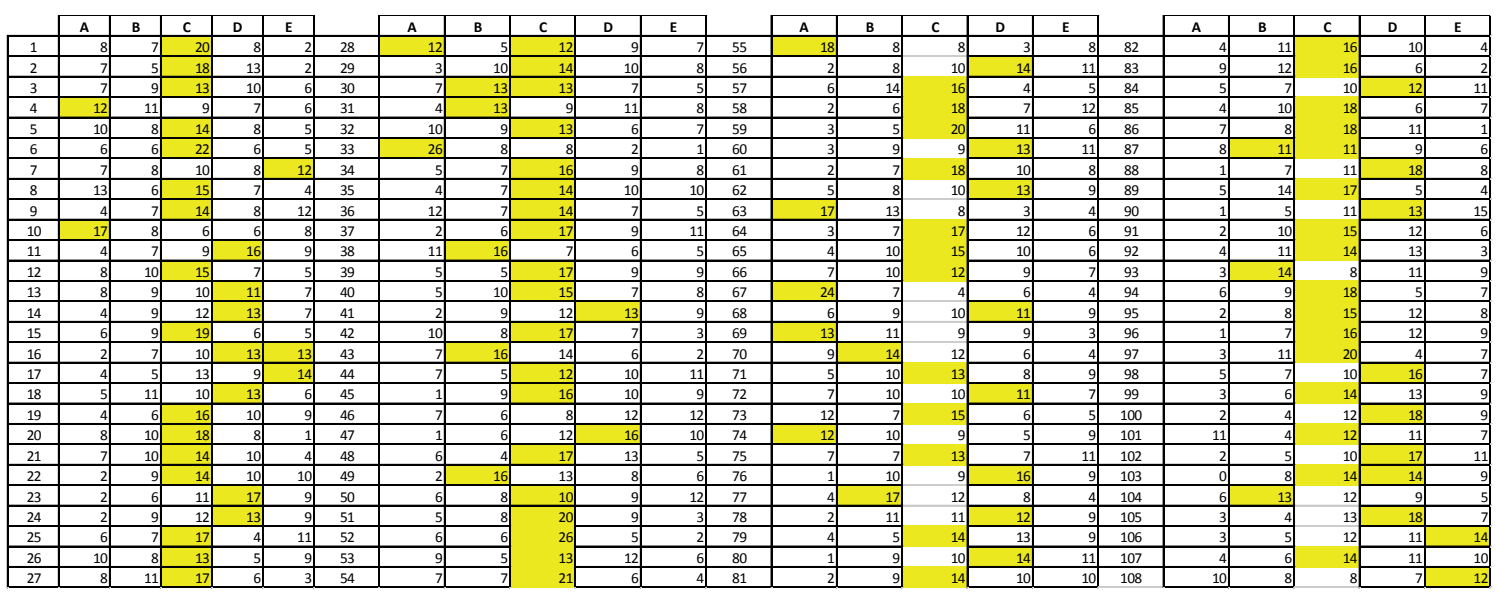

Fuente: Investigación propia.

Cuadro 2. Cuadro estadístico de recopilación de datos basados en baremos del CTI

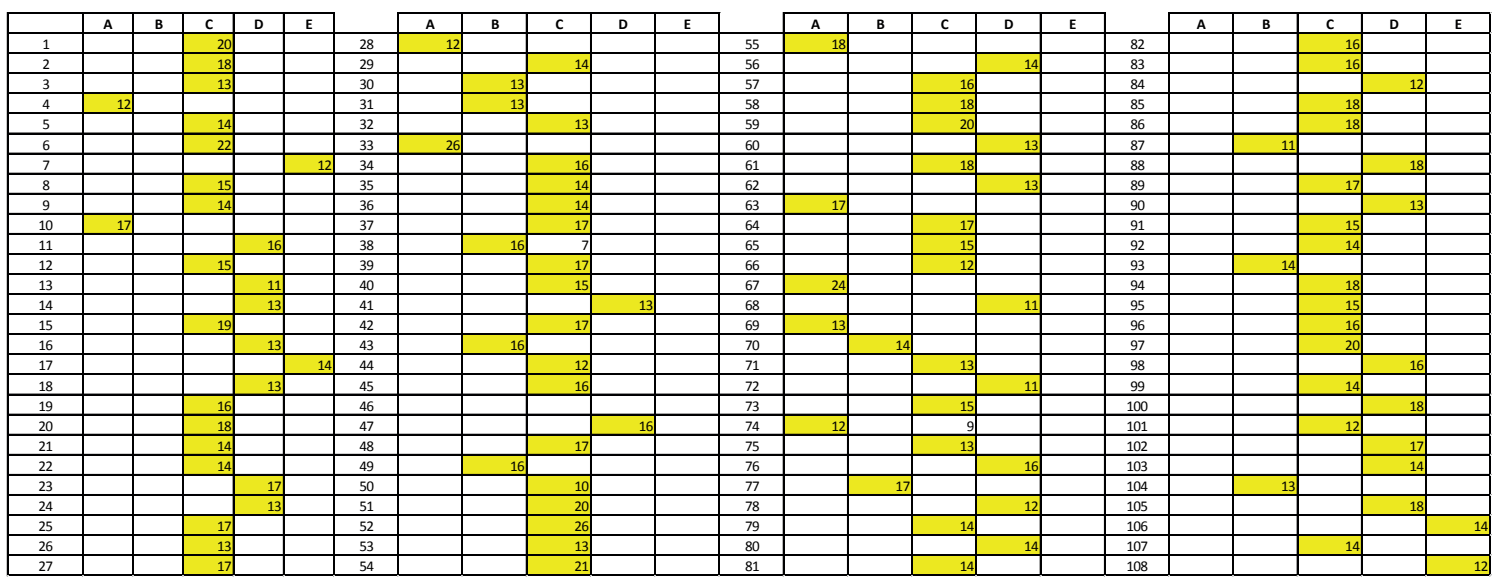

Fuente: Investigación propia.

Cuadro 3. Perfil general pensamiento constructivo colegio John Davis Rockefeller

\begin{tabular}{|l|c|c|}
\hline \multicolumn{1}{|c|}{ COMPETENCIAS } & PD & T \\
\hline Pensamiento constructivo & 97 & 41 \\
\hline Emotividad & 80 & 42 \\
\hline Motivación & 21 & 38 \\
\hline Tolerancia & 10 & 35 \\
\hline impasibilidad & 24 & 48 \\
\hline Capacidad de sobreponerse & 18 & 43 \\
\hline
\end{tabular}




\begin{tabular}{|l|c|c|}
\hline Eficacia & 41 & 30 \\
\hline Pensamiento positivo & 14 & 47 \\
\hline Actividad & 22 & 37 \\
\hline Responsabilidad & 23 & 57 \\
\hline Pensamiento superticioso & 18 & 66 \\
\hline Rigidez & 51 & 62 \\
\hline Extremismo & 19 & 63 \\
\hline Suspicacia & 17 & 67 \\
\hline Intolerancia & 13 & 62 \\
\hline Pensamiento esotérico & 40 & 64 \\
\hline Creencias paranormales & 20 & 61 \\
\hline Creencias irracionales & 21 & 51 \\
\hline Ilusión & 50 & 58 \\
\hline Euforia & 17 & 38 \\
\hline Pensamiento estereotipado & 6 & 57 \\
\hline Ingenuidad & 26 & 65 \\
\hline Deseabilidad & 30 & \\
\hline Validez & 32 & \\
\hline
\end{tabular}

Fuente: Investigación propia.

Figura 1: Perfil general del pensamiento constructivo del colegio John Davis Rockefeller
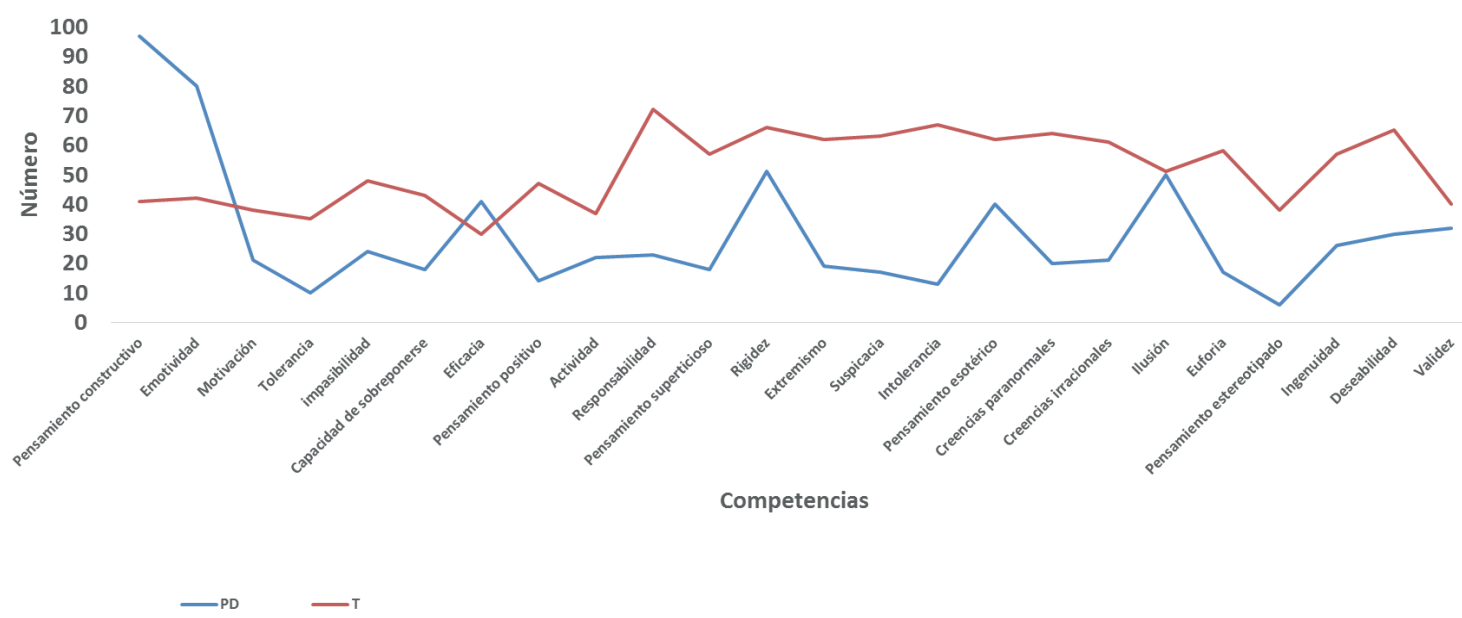

Fuente: Investigación propia 
Figura 2: Perfil general pensamiento constructivo colegio John Davis Rockefeller

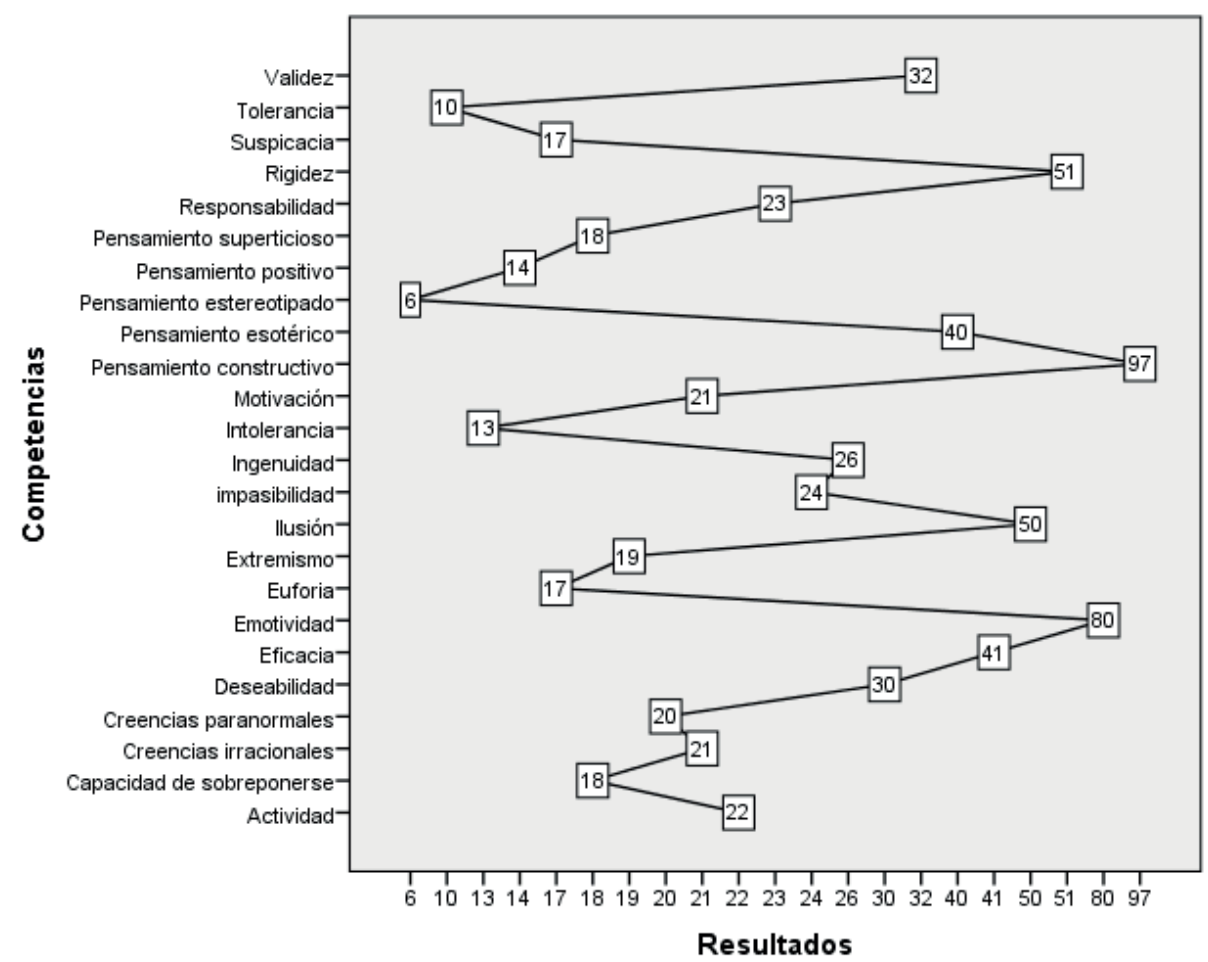

Fuente: Investigación propia

En el cuadro 3, a través de la aplicación del programa estadístico SPSS, se muestra el perfil de la población estudiada, se aprecia que en este entorno la responsabilidad tiene un valor de 23 puntos cuando debería ser 72 como puntos idóneos, con este puntaje se puede determinar que los estudiantes de educación media, aún no han desarrollado esta competencia, que se relaciona con la orientación a la acción y a la planificación con un alto grado de reflexión.

Otro de los puntos que se aprecia en el análisis del entorno y que debe sujetarse a grandes cambios es el pensamiento positivo ligado a la euforia y autoestima, estos

\section{Conclusiones}

La investigación ayudó a comprender lo que significa el Pensamiento Constructivo CTI y demostrar qué tan efectivo resulta su aplica- tres deben estar en conjunción, cuando uno sube, por ejemplo el pensamiento positivo y la euforia están en los mismos niveles; se dice que la persona está lista para el cambio y por ende su autoestima se encuentra en niveles neutrales, según los puntos obtenidos, los estudiantes deben prepararse más para producir cambios verdaderamente radicales, el pensamiento positivo tiene 14 puntos cuando debe superar los 47 , la euforia posee 17 cuando debería tener 58, y la autoestima es 21 cuando debería poseer 380 superar este nivel, para modificar este comportamiento el cambio debe ser realmente motivador. 
de emprendimiento, llegando a la conclusión de que la mayor cantidad de estudiantes involucran la parte emocional como un factor de mucha importancia para tener éxito en la consecución de sus proyectos.

Los resultados generales al aplicar el CTI a los estudiantes del colegio John Davis Rockefeller, demostraron la falta de positivismo y responsabilidad al momento de emprender, careciendo de una cultura de emprendimiento en la institución educativa, uno de los puntos más débiles se refiere al factor pensamiento positivo, este punto es de vital importancia y se debería trabajar más en desarrollar en los estudiantes que inician su proceso de emprendimiento, ya que este es el motor del bienestar personal y profesional, condiciones necesarias para convertirse en emprendedor, tomando en cuenta que una puntuación baja en esta competencia trae como consecuencias que el emprendedor postergue las tareas difíciles o desagradables y tienda a abandonar o desanimarse ante los fracasos, en cambio una puntuación alta en este factor la persona trata de poner en práctica acciones

\section{Referencias}

Álvarez, R., \& Camargo, C. (2007). Pensamiento constructivo y variables sociodemográficas en estudiantes de primero a trecer semestre de la Facultad de Psicología de la Universidad Autónoma de Bucaramanga. Psicología desde el Caribe.

Chavéz Becerra, M., Contreras Gutiérrez, 0., \& Velásquez Ortiz, M. (2013). Adaptación y pensamiento constructivo en estudiantes universitarios. Universidad Nacional Autonoma de México, 16(30), 311-323.

Contreras, G., Chávez, B., Aragón, B., \& Velásquez, O. (2011). Estrategias de pensamiento constructivo en estudiantes universitarios. Universitas Psychologica 10(1), 99-111.

CTI. (2015). TEST-GRATIS.com. Obtenido de http://www.tests-gratis.com/tests_inteligencia_emocional/test-cti-inventario-depensamiento-constructivo.htm efectivas para solucionar problemas, les gusta los desafíos e intentan aprender de la experiencia; la motivación debe intervenir como un componente importante en el emprendimiento, este dará lugar a conductas motoras y a cada estado de ánimo le corresponde una manifestación conductual; la capacidad de sobreponerse está enfocada a la resistencia que ponen las personas al volver a su estado inicial, es la resistencia que se pone al crecer en situaciones adversas o de presión en el proceso experiencial.

Con estos resultados podemos concluir que es necesario generar una cultura orientada al emprendimiento en los estudiantes mediante la aplicación de metodologías creativas e innovadoras para la enseñanza en temas de emprendimiento teórico-práctico y vivenciales, que simulen situaciones positivas y negativas en el proceso de emprendimiento, así como en mejorar el comportamiento conductual y emocional de los estudiantes para mejorar la actitud y compromiso hacia la consecución de sus proyectos de vida.

De la Fuente Arias, J., Vera Martínez, M. M., \& Cardelle-Elawar, M. (2012). Aportaciones de la Psicología de la innovación y del Emprendimiento a la Educación, en la Sociedad del Conocimiento. Education \& Sychology, 10(3), 941-966.

Epstein, S. (1994). Integration of de cognitive and psychodynamic unconsciois. American Psycologis, 49(8), 709-724.

Epstein, S. (1998). Constructive Thinking: The key to Emotinal Intelligence. Westport: Preaeger Publishers.

Epstein, S. (2012). Inventario de pensamiento constructivo. Madrid: TEA.

García, H. D. (2005). El pensamiento constructivo y su relación con la visión de sí mismo del mundo y del futuro en ingresantes de la facultad de ciencias humanas de San Luis. Fundamentos en Humanidades, VI(11), 199-219. 
Gerencie.com. (28 de abril de 2018). Obtenido de https://www.gerencie.com/emprendimiento.html: https://www.gerencie.com/ emprendimiento.html

Gutiérrez, J. A., Asprilla, M, E., \& Gutiérrez, J. (2016). Estado del arte del emprendimiento empresarial en materia de ciencia, tecnología e innovación. Revista universitaria RUTA 18(2).

Hernández, I., \& Alvarado, J. C. (2014). Creatividad e innovación: competencias genéricas o transversales en la formación profesional. Revista Virtual Universidad Católica del Norte, 44 (febrero-mayo), 137-151.

Hernández, C., \& Arano, R. (2015). El desarrollo de la cultura emprendedora en estudiantes universitarios para el fortalecimiento de la visión empresarial. Ciencias Administrativas, 28-37.

Huerta, E. (2019). Educación emocional en clave de integración. Una aportación a la innovación educativa. Revista Panamericana de Pedagogía, 27, 169-192.

López Sánchez, M., Jiménez Torres, M., \& López Núñez, J. (2011). Análisis de la relación entre inteligencia emocional y bienestar psicológico en el profesorado de Educación Infantil y Primaria. Ciencias de la Educación, 228(1), 439-452.

Marulanda Montoya, A., Correra Calle, G., \& Mejía Mejía, F. (2009). Emprendimiento:Visiones desde las teorias del comportamiento humano. Revista Escuela de Administración de Negocios, 66, 153-168.
Muñoz, A. (4 de marzo de 2010). Cepvi. Obtenido de http://www.cepvi.com/index.php/psicologia/articulos/pensamiento-constructivo-e-inteligencia-emocional? start=10: http://www.cepvi.com/index.php/psicologia/articulos/pensamiento-constructivoe-inteligencia-emocional?start $=10$

Pelta, R. (2009). Desing Thinking. Catalunya: Universidad Oberta de Catalunya.

Puebla, I., \& Termiño, A. (2018). La importancia de los Centros de Simulación Empresarial en la formación Universitaria. International Journal of Information Systems and Software Engineering for Big Companies (IJISEBC), 5(2), 115-122.

Rodríguez Ramírez, N. E., Delgadillo Salgado, M. D., \& Torres Trejo, S. L. (2018). Los ambientes de aprendizaje constructivistas como alternativa para generar innovación en la universidad International. International Journal of Information Systems and Software Engineering for Big Companies (IJISEBC), 5(2), 41-52.

Salovey, P., \& Mayer, J. (1990). Emotinal intelligence. Imagination, Cognition and Personality, 9(3), 185-211.

Toca, A. (2017). Obtenido de https://www.excelsior.com.mx/opinion/antonio-toca/ 2017/03/11/1151394https://www.excel sior.com.mx/opinion/antonio-toca/ 2017/03/11/1151394

Zamora, B. C. (2018). La importancia del emprendimiento en la economía: el caso de Ecuador. Espacios, 39(07), 15. 